\title{
Hippokratische Medizin (Cinquième Colloque International Hippocratique), ein internationales Kolloquium in Berlin
}

Von Huldrych Koelbing

Mit Hippokrates von Kos (460-377 v. Chr.) beginnt die wissenschaftliche Medizin. Die CEuvres d'Hippocrate, die Emile Littré um die Mitte des letzten Jahrhunderts in griechischer und französischer Sprache herausgab, umfassen 10 Bände; welche der rund 60 einzelnen Werke aber von dem großen Meister selbst stammen, läßt sich nicht mit Sicherheit ausmachen. Die einfachste Antwort lautet, gar keines - aber sie ist sicher falsch. Unabhängig von diesen Fragen um die Verfasserschaft besitzen wir jedenfalls in der Hippokratischen Schriftensammlung eine ungemein reiche Dokumentation griechischen medizinischen Denkens und ärztlicher Beobachtung, verbunden mit Reflexionen über Möglichkeiten, Aufgaben und Grenzen des ärztlichen Handelns. Das Corpus Hippocraticum ist zudem die einzige große Sammlung griechischer Fachprosa der klassischen Zeit, die erhalten geblieben ist. Immer aufs neue befassen sich deshalb Philologen, Philosophen, Historiker und Mediziner damit, jeder von seinem besondern Gesichtspunkt aus. Als vor 12 Jahren die beiden Straßburger Graezisten Louis Bourgey und Jacques Jouanna zwei Dutzend dieser Hippocratisants zusammenriefen, ging es ihnen darum, durch Austausch von Wissen und Ansichten der Spezialisten zu einem umfassenden Verständnis der Hippokratischen Überlieferung und ihres Nachwirkens in der späteren Medizin beizutragen. Jenem ersten Hippokratischen Kolloquium sind in dreijährigen Abständen vier weitere gefolgt, zuerst im französischen Sprachgebiet - Mons (Belgien), Paris, Lausanne * - und nun, vom 10. bis 15. September 1984, in Berlin. Das «Cinquième Colloque International Hippocratique» ließ die übliche Entwicklung solcher Unternehmungen erkennen: aus dem Gruppengespräch ist ein Kongreß geworden, mit rund 90 Teilnehmern aus fünfzehn Ländern, von Finnland und Rumänien bis USA und Kanada. Die Mehrzahl stammte freilich aus dem französischen, italienischen oder deutschen Sprachgebiet,

\footnotetext{
* François Lasserre und Philippe Mudry (Herausgeber), Formes de pensée dans la Collection Hippocratique, Actes du IV Colloque International Hippocratique (Lausanne, 21-26 septembre 1981), Librairie Droz, Genève 1983.
} 
einschließlich Ost-Berlin. Für den reibungslosen Ablauf im hochmodernen Klinikum Steglitz sorgten Prof.Rolf Winau und seine Mitarbeiter vom Institut für Geschichte der Medizin an der Freien Universität Berlin; der Berliner Senator für Kunst und Wissenschaft kam großzügig für die Organisationskosten auf, und sein Vertreter, Senatsrat Hensch, betonte auf einem abendlichen Empfang, daß das heutige Berlin seine internationale Geltung auf geistigem Gebiet suche.

Der Präsident der Freien Universität Berlin, der Jurist Dieter Heckelmann, äußerte in seinem Grußwort die Hoffnung, die Beschäftigung mit Hippokrates und seiner Auffassung der Medizin könne vielleicht zur Überwindung der Krise in der heutigen Medizin mit ihrer unüberschaubar werdenden spezialistischen Zersplitterung beitragen. Der Schimmer dieser Hoffnung motiviert wohl auch den Medizinhistoriker in seiner Lehrtätigkeit, auch wenn er sich keinen Illusionen über die sehr beschränkten Möglichkeiten seines Einflusses hingibt.

Ein großer Teil der mehr als dreißig Referentinnen und Referenten legten indessen - wie könnte es anders sein? - Ergebnisse ihrer Spezialstudien vor. Als Hauptthema hatten die Organisatoren die Hippokratischen EpidemienBücher aufs Programm gesetzt, jene sieben Bücher, in denen uns konkrete ärtliche Beobachtungen und daraus abgeleitete aphoristische Lehrsätze überliefert sind. Die beiden ältesten (1 und 3) hat wohl der große Hippokrates von Kos um 410 v. Chr. selbst redigiert, die übrigen fünf stammen von uns unbekannten Autoren aus dem Anfang $(2,4,6)$ und der Mitte $(5,7)$ des 4. Jahrhunderts (Diller). Die vom heutigen Sprachgebrauch abweichende Bezeichnung Epidêmiai ergab sich daraus, daß Hippokrates die bei der Bevölkerung eines bestimmten Ortes in einem gegebenen Zeitraum aufgetretenen Erkrankungen beschrieb; die späteren Bücher enthalten aber zahlreiche individuelle und für sich stehende Krankengeschichten. Das Faszinierende daran ist für Fernand Robert (Paris), den Nestor des Kolloquiums, die Lebensnähe der Beobachtungen, die hier in der Sprache des Alltags mitgeteilt werden. In den Epidemien-Büchern 5 und 7 sieht Robert eine Equipe von Ärzten am Werk, die sich um eine kritische Beurteilung ihres eigenen Tuns bemühten - ein wesentliches Element wissenschaftlicher Medizin.

Mit der Umwandlung der individuell oft so variablen Krankenbeobachtung in die allgemeingültige Regel, die im Aphorismus festgehalten, gelehrt und gelernt wird, befaßten sich mehrere Beiträge (Jutta Kollesch, OstBerlin; Volker Langholf, Hamburg; Amneris Roselli, Cosenza); Frau Roselli fand, daß diese Lehrsätze die Tendenz zeigen, sich von der ärztlichen Praxis 
zu entfernen, um sich den schriftlich überlieferten Lehren anzupassen. Auf eine bemerkenswerte Diskrepanz zwischen Absicht und Ausführung beim Niederschreiben klinischer Beobachtungen ist Gheorge Bratescu (Bukarest) gestoßen: grundsätzlich sieht Hippokrates in Epidemien 1 und 3 das örtliche Krankheitsspektrum in Abhängigkeit vom Wetterverlauf des Jahres; bei der Wiedergabe der einzelnen Krankengeschichten ist jedoch vom Einfluß der Witterung auf den Krankheitsverlauf überhaupt nicht mehr die Rede.

Ein neues Moment bringt der Computer in die philologische HippokratesForschung; auch hier wird nun eifrig quantifiziert. Grundlage für Arbeiten in dieser Richtung ist vor allem die Concordance Hippocratique, die unter der Leitung von Gilles Maloney in Québec entstanden ist und die die insgesamt 367859 Wörter des Corpus Hippocraticum in ihrem jeweiligen Textzusammenhang auflistet. Maloney selbst legte am Berliner Kolloquium aufgrund einer numerischen Textanalyse dar, daß die Ärzte der Epidemien bei ihren Verordnungen den Zerealien und dem Gemüse den Vorzug gaben vor dem Wein und den damit hergestellten Medikamenten, die in den übrigen Hippokratischen Schriften besonders beliebt sind. Jacques Jouanna, der jetzt an der Sorbonne lehrt, wendet dagegen die Zählung der in verschiedenen Texten vorkommenden Ausdrücke an, um ihre Autoren zu identifizieren und zu unterscheiden. Aufgrund dieser terminologischen Analysen, die sich in imposanten Tabellen niederschlagen, kommt er beispielsweise zum Schluß, der Verfasser der Epidemienbücher 1 und 3 könne nicht auch das als Prognôstikon bekannte kurze Lehrbuch der Krankenuntersuchung geschrieben haben, das uns doch nach Inhalt und Geist ebenfalls als ein Werk des Hippokrates selbst imponiert. Die Vielgestaltigkeit der Hippokratischen Schriften wird jedenfalls auch durch den Computer sehr deutlich gemacht.

Auf geistesgeschichtliche Zusammenhänge ging Charles Lichtenthaeler (Hamburg/Lausanne) ein, indem er den hippokratisch-wissenschaftlichen Begriff der Krankheitsmaterie (materia peccans), die vom Körper, unter Umständen mit Hilfe der Medizin, verarbeitet und ausgeschieden werden muß, mit alten magischen Vorstellungen in Verbindung brachte. Joseph Alsina (Barcelona) beleuchtete die vieldiskutierte Beschreibung der sog. Pest von Athen (430/29), einer wirklichen Epidemie im modernen Sinn des Wortes, durch den Historiker Thukydides unter einem neuen Gesichtspunkt: er sieht darin weniger eine Krankheitsbeschreibung im Hippokratischen Sinn als die Darstellung einer Tragödie, so wie sie Sophokles im König Oedipus gestaltete. 
Als Medizinhistoriker hob der Verfasser dieses Berichtes das Widerspiel von Optimismus und Zurückhaltung in der Therapie der hippokratischen Ärzte hervor: grundsätzlich sind die Krankheiten für Hippokrates und seine Schüler als Naturvorgänge erforschbar und lenkbar; Gelenksverrenkungen und Knochenbrüche müssen sich den unerbittlichen Kräften der Mechanik fügen; aber manche Krankengeschichten und Lehrsätze mahnen zur Vorsicht bei der ärztlichen Behandlung. «Nützen oder doch wenigstens nicht schaden» bleibt seit Hippokrates (Epidemien 1, Kap.11) die Aufgabe des Arztes.

Hippokratisches Denken hat noch zur Zeit der Aufklärung die wissenschaftlichen Diskussionen beeinflußt. Dies machte Gerhard Rudolph (Kiel) am Beispiel des Mittagsschlafes deutlich, dessen Schädlichkeit lange geradezu Dogma war. Hippokrates ist zuweilen auch zu Unrecht als Kronzeuge für die eigene Lehrmeinung in Anspruch genommen worden, so von den Vitalisten der Schule von Montpellier im 18. Jahrhundert, für die das Leben an ein besonderes "principe vital» gebunden war; Antoine Thivel (Nice) weist jedoch nach, daß die Hippokratiker zur Erklärung der Lebensprozesse eher materielle Vorgänge heranzogen. Man könnte natürlich darüber streiten, ob die «eingeborene Wärme», die nach hippokratischer Auffassung den Organismus im Gange hält, tatsächlich ein physikalisch-materialistischer Begriff ist; Lichtenthaeler sieht auch darin ein magisches Relikt.

Das Nachwirken Hippokratischer Lehren trat überhaupt in Berlin als zweites großes Thema neben das enger gefaßte der Epidemien-Bücher, und das mit Recht. Von den Einzelvorträgen, die darauf eingingen, seien nur noch zwei erwähnt: Innocenzo Mazzini (Macerata) sprach über lateinische Renaissance-Übersetzungen der Epidemien, Giuliana Lanata (Genova) über hippokratische Einflüsse im römischen Recht. Der letzte Verhandlungstag bot eine eindrücklich geschlossene Reihe von Vorträgen zu diesem Fortwirken, nach Epochen gegliedert: Hellenismus (Fridolf Kudlien, Kiel), arabische Medizin (Ursula Weisser, Erlangen), europäisches Mittelalter (Gerhard Baader, Berlin; Gundolf Keil, Würzburg), Renaissance (Vivian Nutton, London). Man darf sich darauf freuen, gerade diesen Teil des Cinquième Colloque International Hippocratique im vollständigen Verhandlungsbericht, hoffentlich noch dieses Jahr, lesen zu können. 


\section{Summary}

This is a report on the 5th International Hippocratic Colloquium, held in September 1984 in Berlin (West). The main subject of discussion were the seven books of Epidemics, containing case histories collected at different times in different places and written down by at least three authors or groups of authors. Some speakers stressed the self-criticism of these physicians, others emphasized their difficulties in transforming their bed-side experience into general rules of prognosis and treatment. The value of computer-assisted quantitative analysis of texts remained controversial.-An important group of papers were devoted to the historical influence of Hippocratic writings down to the 18th century.-The 6th International Hippocratic Colloquium is to be held in 1987 in Canada (Université de Québec).

Prof. Dr. med. H. M. Koelbing

Medizinhistorisches Institut der Universität Zürich

Rämistraße 71

CH-8006 Zürich 